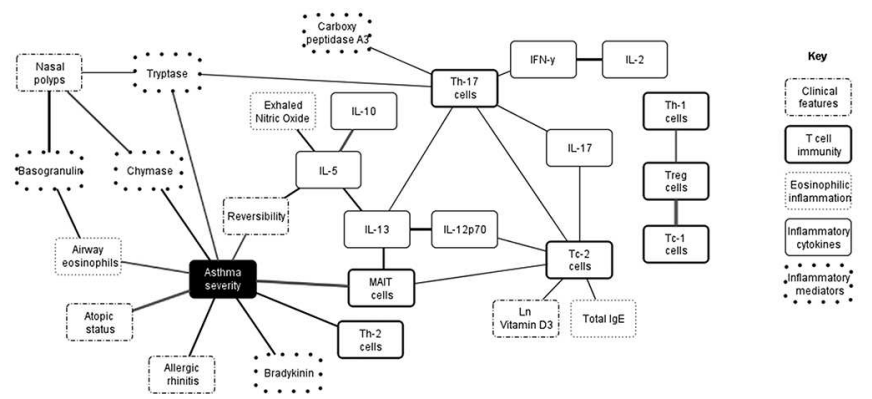

Abstract S23 Figure 1. Bayesian Network Analysis showing statistically significant relationships between clinical and immunological parameters measured in the study. The strength of the interactions (as Euclidean distance) is shown by line weight. Figure 1. Bayesian Network Analysis showing statistically significant relationships between clinical and immunological parameters measured in the study. The strength of the interactions (as Euclidean distance) is shown by line weight.

Introduction and Objectives Asthma is a complex disease involving many cell types including CD4+ and CD8+ T cells, eosinophils, basophils and mast cells, and their soluble mediators. Poor understanding of disease heterogeneity and mechanisms underlying distinct clinico-pathological endotypes limits progress.

We hypothesised that identification of distinct multidimensional endotypes using combined clinical and pathological parameters would enable a better understanding of asthma mechanisms and phenotypes. We undertook to analyse a range of cell types and mediators across a wide clinical spectrum of asthma and health with application of a novel analytical approach: topological data analysis (TDA).

Methods 76 adult subjects underwent extensive phenotyping, using clinical assessment, lung function, methacholine challenge, sputum induction, phlebotomy, and bronchoscopy. Samples were analysed for measurement of CD4+, CD8+ and invariant $\mathrm{T}$ cell subsets, of $\mathrm{T}$ cell cytokines, of mast cell and basophil mediators, bradykinin and vitamin D, using 9-colour flow-cytometry, RTPCR, multiplex ELISA and mass spectrometry. We used Bayesian network analysis (BNA) to define the inter-connectivity between the key biological parameters investigated and TDA to identify novel multi-dimensional endotypes of asthma.

Results We generated a Bayesian belief network using 37 key parameters on a subset of 62 subjects with the most complete data (figure), revealing asthma severity as a multidimensional feature, influenced by a wide variety of parameters, including Th2 inflammation, mast cells and basophils.

A TDA network generated using only pathological features revealed distinct pathological endotypes including i) mast cell / basophil mediated ii) severe, obese, high bradykinin iii) minimal inflammation, without allergic rhinitis iv) Th2 mediated, milder disease. Significant differences were observed between clusters in carboxypeptidase, bradykinin, basogranulin, Th2 cells, and IL-4,-5,-12p70.
A TDA network generated using only clinical features revealed a clear dichotomy between steroid responsive and steroid refractory disease. Differences were observed between clinical groups in tryptase, chymase, eosinophils and Th2 and mucosal associated invariant T (MAIT) cells.

Conclusions This study highlights the diversity of aberrant immune responses associated with different clinical and pathological phenotypes of asthma. Attention should focus on further defining these distinct subtypes using such unbiased techniques and characterising under-researched pathways such as CD8 $+\mathrm{T}$ cells, basophils and bradykinin.

\section{Clinical trials in COPD}

\section{S24 A SELF-MANAGEMENT PROGRAMME OF ACTIVITY, COPING AND EDUCATION (SPACE) FOR COPD: A RANDOMISED NON-INFERIORITY TRIAL IN A PULMONARY REHABILITATION POPULATION}

E Horton ${ }^{1}, K$ Mitchell ${ }^{2}, V$ Johnson-Warrington ${ }^{2}, L$ Apps $^{2}, H_{\text {Young }}{ }^{2}$, S Singh ${ }^{1}, S$ Singh ${ }^{2}$.; ${ }^{1}$ Coventry University, Coventry, United Kingdom; ${ }^{2}$ Centre for Exercise and Rehabilitation Science, University Hospitals Leicester NHS Trust, Leicester, United Kingdom

\subsection{6/thoraxjnl-2013-204457.31}

Introduction Self-managed programmes for patients with COPD offer an alternative to conventional pulmonary rehabilitation (PR). Previously reported self management (SM) programmes either offer short education sessions or action plans or are long intensive programme with a high level of support which are more comprehensive than tradition UK PR programmes. There is limited data reported on self managed approaches to PR which offer minimal support for education, skills acquisition and exercise in comparison to PR in the UK. We have recently developed a SM programme for patients with COPD (SPACE for COPD) and have completed studies in primary care ${ }^{1}$. The demand for PR is high but the service is compromised by limited access and drop out rates. Therefore the aim of this study was to determine if SPACE for COPD is noninferior to (as good as) PR for patients with COPD over 7 weeks.

Methods 287 patients (187 male: mean (SD) age 67 (9) yrs; $\mathrm{FEV}_{1} 1.25$ (0.55); BMI $27.63(6.22) \mathrm{kg} / \mathrm{m}^{2}$ ) with COPD were consented and randomised to either SPACE for COPD or PR. Those who were assigned to the SPACE for COPD group received an introduction to the SPACE for COPD manual and 2 telephone calls at 2 and 4 weeks. Those assigned to PR received 14, 2 hour sessions of education and exercise over 7 weeks. The primary outcome was the Chronic Respiratory Questionnaire (CRQ) dyspnoea, with secondary measure of CRQ fatigue, emotion and mastery, Incremental Shuttle Walk Test (ISWT) and Endurance Shuttle Walk Test (ESWT). Measures were taken at

\begin{tabular}{|c|c|c|c|c|c|c|c|}
\hline & \multicolumn{3}{|c|}{ SPACE for $C O P D n=94$} & \multicolumn{3}{|c|}{ Pulmonary Rehabilitationn=84 } & \multirow{2}{*}{$\begin{array}{l}\text { Between group differences } \\
(95 \% \mathrm{Cl})\end{array}$} \\
\hline & Baseline (SD) & 7 weeks (SD) & Change $(95 \% \mathrm{Cl})$ & Baseline (SD) & 7 weeks (SD) & Change $(95 \% \mathrm{Cl})$ & \\
\hline Dyspnoea & $2.58(0.93)$ & $3.11(1.23)$ & $0.55(0.26$ to 0.85$) p=<0.001$ & $2.42(0.91)$ & $3.38(1.18)$ & $0.87(0.61$ to 1.13$) p<0.001$ & $-0.32(-0.71$ to 0.08$) p=0.113$ \\
\hline Fatigue & $3.42(1.19)$ & $3.71(1.22)$ & $0.13(-0.14$ to 0.39$) p=0.339$ & $3.36(1.20)$ & $4.09(1.49)$ & $0.54(0.27$ to 0.82$) p<0.001$ & $-0.41(-0.79$ to 0.03$) p=0.033$ \\
\hline Emotion & $4.41(1.24)$ & $4.56(1.20)$ & $0.005(-0.23$ to 0.24$) p=0.965$ & $4.37(1.24)$ & $4.92(1.03)$ & $0.53(0.26$ to 0.79$) p<0.001$ & $-0.52(-0.88$ to 0.17$) p=0.004$ \\
\hline Mastery & $4.50(1.40)$ & $4.78(1.31)$ & $0.15(-0.11$ to 0.40$) p=0.259$ & $4.36(1.30)$ & $4.94(1.19)$ & $0.58(0.34$ to 0.81$) p<0.001$ & $-0.43(-0.77$ to 0.09$) p=0.014$ \\
\hline ISWT (metres) & $260.24(147.91)$ & $277.86(145.59)$ & $17.62(3.26$ to 31.98$) \mathrm{P}=0.17$ & $268.61(149.89)$ & $310.13(156.46)$ & $41.52(23.91$ to 59.12$)$ & $-23.90(-46.33$ to -1.47$) p=0.038$ \\
\hline ESWT (seconds) & $231.42(231.00)$ & 444.33 (393.09) & $212.91(139.49$ to 286.34$) \mathrm{P}<0.001$ & $189.14(96.25)$ & 534.85 (395.38) & $345.70(260.20$ to 431.20$) p<0.001$ & $-132.78(-244.63$ to -20.94$) p=0.02$ \\
\hline
\end{tabular}


baseline and 7 weeks. Within and between group differences were analysed using paired and unpaired t-tests respectively.

Results Please see table 1.

Conclusion SPACE for COPD can improve dyspnoea and endurance capacity over 7 weeks to a similar level to PR, although it remains unclear to its noniferiority to PR. The SPACE for COPD programme does offer a number of health benefits despite it involving limited support and could offer a suitable alternative to patients with COPD who would otherwise not attend conventional rehabilitation.

\section{REFERENCES}

1. Mitchell-Wagg K et al. (2012). Thorax 67 (Suppl_2) A25-26.

\section{S25 THE EFFECTS OF MAINTENANCE SCHEDULES FOLLOWING PULMONARY REHABILITATION IN PATIENTS WITH CHRONIC OBSTRUCTIVE PULMONARY DISEASE}

${ }^{1} \mathrm{P}$ Browne, ${ }^{1} \mathrm{~S}$ Olive, ${ }^{2} \mathrm{~L}$ Staunton, ${ }^{1} \mathrm{~A}$ Clark, ${ }^{3} \mathrm{E}$ Wilson, ${ }^{1} \mathrm{P}$ Galey, ${ }^{1} \mathrm{E}$ Knights, ${ }^{1} \mathrm{H}$ Woodhouse, ${ }^{1} \mathrm{~S}$ Robinson, ${ }^{3} \mathrm{AM}$ Wilson; ${ }^{1}$ Norfolk and Norwich University Hospital NHS Foundation Trust, Norwich, UK; ${ }^{2}$ Norfolk Community Health and Care NHS Trust, Norwich, UK; ${ }^{3}$ University of East Anglia, Norwich, UK

\subsection{6/thoraxjnl-2013-204457.32}

Background There is good evidence that pulmonary rehabilitation (PR) provides benefit for patients with chronic obstructive pulmonary disease (COPD) in terms of quality of life and daily functioning. However it is generally accepted that the benefits diminish over time.

Methods We conducted a randomised controlled parallel study of a maintenance programme, following standard PR, consisting of a two hour session of education and strength and endurance training every 3 months versus standard care. Measurements were made, at baseline (prior to a standard PR programme), at randomisation (after successful completion of a PR programme) and after 12 months, of the chronic respiratory questionnaire (CRQ), endurance shuttle walk test (ESWT), EuroQol (EQ5D), hospital anxiety and depression score (HADS) and activity questionnaires. CRQ was also completed every 3 months by post.

Results 250 (139 male) patients, mean (SD) age of 69.2 (9.2) years, FEV1 $41(16) \%$ predicted, provided informed consent to participate in the study. The mean $(95 \% \mathrm{CI})$ improvement in CRQ following the initial PR was $0.76(0.59,0.93)$ units. 148 patients entered the randomised part of the study. There remained a significant improvement in CRQ dyspnoea at 12 months compared to baseline for the group as a whole. However, there was no statistically significant differences detected between the intervention and control groups for the CRQ dyspnoea score, which amounted to $0.19(-0.26,0.64)$ units, or other domains of the CRQ. There was no difference in the ESTW distance between the two groups (109.1 (-100.1 to 318.2) metres) or HADS $(-0.2(-2.41,2)$ units). There was a higher level of selfreported activity, according to the visual analogue score of 16.2 / 100 , in the maintenance group but not the reported metabolic equivalent (MET)-minutes per week. There was no difference in any of the CRQ measures at any of 3 monthly measurements between the intervention and control groups

Conclusion A maintenance programme of 3monthly 2 hour sessions does not improve outcomes in patients with COPD after 12 months. We cannot recommend that our maintenance programme is adopted. It is likely that a maintenance programme should commence earlier than 3 months and possibly be more intensive.
S26

SEVERE HOSPITALISED EXACERBATIONS OF COPD WITH AN EOSINOPHILIC PHENOTYPE HAVE FAVOURABLE OUTCOMES WITH PREDNISOLONE THERAPY: SUB-ANALYSIS FROM A PROSPECTIVE MULTI-CENTRE RANDOMISED CONTROL TRIAL

${ }^{1} \mathrm{M}$ Bafadhel, ${ }^{2} \mathrm{NJ}$ Greening, ${ }^{2} \mathrm{~T}$ Harvey-Dunston, ${ }^{2} \mathrm{~J}$ Williams, ${ }^{2} \mathrm{MDL}$ Morgan, ${ }^{3} \mathrm{~F}$ Hussain, ${ }^{1} \mathrm{Ld}$ Pavord, ${ }^{2} \mathrm{~S} J$ Singh, ${ }^{2} \mathrm{MC}$ Steiner; ${ }^{1} / \mathrm{LH}$, NIHR Respiratory BRU, Glenfield Hospital, Leicester, UK; ${ }^{2}$ The Centre for Exercise and Rehabilitation Science, Department of Respiratory Medicine, Glenfield Hospital, Leciester, UK; ${ }^{3}$ Kettering General Hospital NHS Foundation Trust, Kettering, UK

10.1136/thoraxjnl-2013-204457.33

Introduction In moderate exacerbations of COPD, patients with the eosinophilic phenotype ( $>2 \%$ of the total leukocyte count) have better outcomes with prednisolone. However, it remains unclear whether patients with severe exacerbations displaying the eosinophilic phenotype have accentuated recovery following corticosteroid therapy compared to non-eosinophilic COPD exacerbations.

Aim Measure the incidence of eosinophilic and non-eosinophilic severe exacerbations of COPD, from a large prospective enhanced recovery multi-centre randomised control trial and investigate severity and recovery between these groups.

Methods COPD patients entering the programme delivered immediately on hospitalisation for an acute exacerbation of chronic respiratory disease to improve long term health outcomes (clinical trial registration ISRCTN05557928) were analysed using admission details, length of stay and proceeding exacerbation history. All patients were dichotomised into eosinophilic $\left(>200 \times 10^{6}\right.$ cells $/ \mathrm{mL}$ and/or $>2 \%$ of the total leukocyte count) and non-eosinophilic. CRP was measured on admission.

Results There were 243 COPD patients (117 males) identified. The mean (range) age was 71 years (45-93) and the majority of patients $(55 \%)$ had been hospitalised for an exacerbation of COPD in the previous 12 months. Of all exacerbations, the inpatient mortality rate was 3\% (median time to death 12 days, range 9-16) and approximately 90\% received both antibiotic and corticosteroid treatment. The incidence of an eosinophilic exacerbation was $25 \%$ (median absolute eosinophil count 100 $\mathrm{x} 10^{6} \mathrm{cells} / \mathrm{ml}$; range 10 to 1500 ). In patients with eosinophilic exacerbations compared to non-eosinophilic exacerbations the median (IQR) CRP concentration was significantly lower $(12 \mathrm{mg} /$ $\mathrm{L}(5-47)$ vs. $55 \mathrm{mg} / \mathrm{L}(18-139), \mathrm{p}<0.001)$; and the presence of an elevated eosinophil count and elevated CRP (>200 x106 eosinophils $/ \mathrm{mL}$ and CRP $>50 \mathrm{mg} / \mathrm{L}$ ) occurred in only $5 \%$ of all exacerbations. The length of stay was significantly shorter in patients with eosinophilic exacerbations compared to non-eosinophilic exacerbations (mean (range) 5.0 (1-19) vs. 6.5 (1-33), $\mathrm{p}=0.015)$. The severity of the index exacerbation or the rate of exacerbations or hospitalisations in the following 12 months was not statistically significant between groups.

Conclusions In severe hospitalised exacerbations of COPD, a proportion have an associated eosinophilic phenotype. These exacerbations are usually not associated with an elevated CRP. Eosinophilic exacerbations have better responses to oral corticosteroids with shortened length of stay.

\section{S27 THE EFFECTS OF STATIN THERAPY ON INFLAMMATORY MARKERS IN PATIENTS WITH COPD: A DOUBLE BLIND RANDOMISED CONTROLLED TRIAL}

${ }^{1} \mathrm{M}$ John, ${ }^{1} \mathrm{AJ}$ Knox, ${ }^{2} \mathrm{TM}$ McKeever, ${ }^{1} \mathrm{G}$ Meakin, ${ }^{1} \mathrm{H}$ Bailey, ${ }^{3} \mathrm{R}$ Cockcroft, ${ }^{3} \mathrm{DJ}$ Shale, ${ }^{1}$ TW Harrison, ${ }^{1} \mathrm{CE}$ Bolton; ${ }^{1}$ Nottingham Respiratory Research Unit, Nottingham, UK; 